\title{
Uso do procedimento de bissecção temporal na avaliação de idosos com doença de Alzheimer: uma revisão sistemática
}

\author{
Using the temporal bisection procedure in the evaluation of \\ elderly with Alzheimer's disease: a systematic review
}

Carla Andreza Almeida Andreatto', Marcelo Bussotti Reyes², Danilla Icassatti Corazza', Renata Valle Pedroso', Flávia Gomes de Melo Coelho1, Sebastião Gobbi', Ruth Ferreira Santos-Galduróz',22

\section{RESUMO}

Objetivo: Realizar revisão sistemática de artigos que utilizaram o método de bissecção, para avaliar a percepção de tempo em idosos com doença de Alzheimer e analisar seus parâmetros. Método: As buscas dos artigos foram conduzidas no período de março a maio de 2011, nas seguintes bases de dados: Web of Science, Science Direct on Line, Biological Abstracts, Psycholnfo e Medline. As palavras-chave e operadores booleanos foram: "interval timing" ou "perception of time" ou "time discrimination" ou "reproduction of time" e "Alzheimer's disease". Também foram realizadas buscas manuais nas referências dos artigos selecionados. Resultados: Quatro artigos contemplavam todos os critérios de inclusão, nos quais foram encontradas grandes variações nos parâmetros utilizados no método. Conclusão: Pacientes com doença de Alzheimer apresentam prejuízos nas tarefas de bissecção de tempo, que podem ser explicados pelo declínio gradual nas habilidades que são utilizadas no teste de percepção de tempo. Há grandes variações nos intervalos de tempo utilizados. Neste contexto, há necessidade de mais estudos, controlados e randomizados, para investigar potenciais efeitos das variações nos intervalos de tempo do método de bissecção. Os resultados de tais estudos poderão contribuir para o estabelecimento de parâmetros mais adequados e fidedignos.

\section{ABSTRACT}

Objective: Perform a systematic review of articles that used the bisection time method to assess the perception of time in patients with Alzheimer's disease by means of the bisection-of-time method, and to analyze its parameters. Method: Searches were conducted from March to May, 2011, in the following databases: Web of Science, Science Direct On Line, Biological Abstracts, Medline and Psycholnfo. Keywords and boolean operators were: "interval timing" or "perception of time" or "time discrimination" or "reproduction of time" and "Alzheimer's disease". Additionally, a manual search was conducted in the references of the selected articles. Results: Four studies fulfilled all inclusion criteria, and large variations in the parameters of the method were found. Conclusion: Patients with Alzheimer's disease

1 Universidade Estadual Paulista (Unesp), Campus de Rio Claro, Instituto de Biociências, Departamento de Educação Física, Laboratório de Atividade Física e Envelhecimento (Lafe), Rio Claro, SP. 


\section{Keywords}

Temporal scales, Alzheimer's disease, perception of time, time interval. show difficulty in performing the task of bisection of time, which can be explained by the gradual decline on the abilities required in the perception of time test. There are large variations regarding the time intervals applied in the method. In such context, controlled-and-randomized future studies are required, in order to investigate the potential effects of time-intervals variations in the bisection-of-time method. The results of such studies would contribute to establish most suitable and reliable parameters.

\section{INTRODUÇÃO}

Muito já foi descrito em relação aos transtornos cognitivos observados na doença de Alzheimer, como prejuízos na memória, atenção, funções executivas e desorientação temporal'. Atualmente, enfatiza-se uma busca por avanços na investigação do funcionamento mental na doença de Alzheimer e, para tentar compreender a desorientação temporal nessa população, um possível avanço seria estudar a capacidade de percepção de tempo.

A percepção de tempo é caracterizada por James² como sendo a duração que um estímulo permanece no campo perceptivo da nossa via sensorial ou, alternativamente, no intervalo entre dois estímulos. A percepção temporal humana é estudada por métodos psicofísicos ${ }^{3}$ e seu processamento neurofisiológico pode ser dividido em quatro escalas temporais, sendo: microssegundos (ecolocalização e localização sonora), milissegundos (coordenação motora, reconhecimento e produção da fala), segundos a minutos (percepção consciente do tempo) e horas do dia (ritmos circadianos)4.

Os métodos empregados para avaliar a percepção de tempo são: estimativa de tempo, discriminação temporal, produção e reprodução de tempo ${ }^{5}$. Os estudos que avaliam a percepção de tempo têm utilizado principalmente tarefas de discriminação temporal ${ }^{6,7}$, nas quais se destaca o método de bissecção temporal, que inclui dois intervalos de tempo (curta duração e longa duração) apresentados como estímulo-padrão. Nessa tarefa, os dois intervalos com variações diferenciadas são apontados ao sujeito, que, por sua vez, deve definir se o tempo do estímulo apresentado é de longa ou de curta duração .

$\mathrm{Na}$ avaliação da percepção de tempo, dois tipos de procedimentos podem ser apresentados: a) os prospectivos, nos quais o sujeito é informado previamente que deverá prestar atenção em um determinado estímulo antes de realizar a tarefa; b) procedimentos retrospectivos, nos quais o sujeito não recebe qualquer informação sobre a tarefa, tendo que determinar o tempo de duração após a apresentação de um estímulo9. Os autores destacam a importância dessa distinção para se determinarem quais domínios cognitivos estariam envolvidos nas diferentes tarefas e apontam um papel fundamental da atenção em tarefas prospectivas e da memória em tarefas retrospectivas.

Os pacientes com doença de Alzheimer apresentam dificuldades em realizar testes que avaliam a percepção de tempo, pois as funções cognitivas que estão comprometidas na demência, como orientação temporal, linguagem, memória, atenção e planejamento (funções executivas), são usualmente necessárias para a realização dos testes ${ }^{10-12}$.

Um dos maiores desafios clínicos é realizar o diagnóstico precoce das doenças neurodegenerativas, entre elas a doença de Alzheimer. A inclusão de uma variável mais específica, como a percepção de tempo, talvez possa ser utilizada para o diagnóstico diferencial e precoce da doença de Alzheimer. Poucos estudos têm analisado a percepção de tempo nessa população e, entre eles, existem divergências entre as características dos métodos utilizados, o que dificulta padronizar ou mesmo elaborar parâmetros que sejam mais adequados para avaliar tal percepção nos pacientes.

Nesse contexto, o objetivo deste estudo foi realizar uma busca sistemática de artigos que utilizaram o método de bissecção para avaliar a percepção de tempo em idosos com doença de Alzheimer, bem como analisar os parâmetros utilizados.

\section{MÉTODOS}

O processo metodológico deste estudo foi constituído por uma revisão sistemática da literatura, orientada pela busca bibliográfica nas seguintes bases de dados: Web of Science, Science Direct on Line, Biological Abstracts, Psycholnfo e Medline. Os operadores booleanos e as palavras-chave utilizados foram: "interval timing" ou "perception of time" ou "time discrimination" ou "reproduction of time" e "Alzheimer's disease". Além da busca nas bases de dados, utilizou-se uma busca manual nas listas de referências dos artigos selecionados. A revisão foi conduzida de março a maio de 2011, e a seleção dos artigos baseou-se nos estudos relacionados aos objetivos da revisão e aos critérios de inclusão e exclusão descritos a seguir. Critérios de inclusão: 1) estudos com indivíduos de idade igual ou acima de 60 anos; 2) diagnóstico de doença 
de Alzheimer no estágio leve; 3) estudos controlados e randomizados; 4) estudos relacionados ao método de bissecção para avaliar percepção de tempo; e 5) idosos que não apresentassem outras comorbidades neurodegenerativas. Critérios de exclusão: 1) estudos de pacientes com doença de Alzheimer nos estágios moderado e avançado; 2) estudos não relacionados ao método de bissecção nas avaliações de percepção de tempo; 3) idosos que apresentassem outras comorbidades neurodegenerativas; e 4) indivíduos com idade inferior a 60 anos.

A busca sistemática dos artigos foi realizada pela autora principal, e a análise deles foi realizada pela autora principal e pelos os demais coautores.

Um resumo das características e resultados dos estudos revisados consta na tabela 1.

\section{RESULTADOS}

A busca bibliográfica resultou, inicialmente, em 70 artigos. Em uma primeira análise, por meio da leitura do título, verificou-se que 46 artigos não se relacionavam com o tema proposto, sendo eles desorientação temporal, atenção sustentada e disfunção neuronal na doença de Alzheimer e na doença de Huntington, restando 24 artigos. Logo após, realizou-se uma leitura dos resumos desses artigos selecionados, sendo verificado que 16 artigos apresentavam a amostra com outras patologias e em modelo ani- mal e quatro artigos não se enquadravam nos critérios de inclusão referentes à metodologia, utilizando métodos como tempo de reação. Restaram, portanto, apenas quatro artigos que se enquadraram no objetivo do estudo e preenchiam todos os critérios de inclusão considerados para a análise.

Os quatro estudos que atenderam aos critérios metodológicos adotados para este trabalho são descritos a seguir.

1) Papagno et al.12 avaliaram a percepção de tempo em uma amostra de 21 pacientes com doença de Alzheimer (média de idade de 71,8 anos; escolaridade média de 8,9 anos) com escore menor que 19 pontos no Miniexame do Estado Mental (MEEM) ${ }^{13}$ e 21 idosos saudáveis (média de idade de 72,2 anos; escolaridade média de 9,1 anos). A percepção de tempo foi avaliada em duas condições de tarefas, e o intervalo consistiu em durações curtas (15 s) e longas (50 s). A primeira condição consistiu em uma tarefa atencional, na qual o sujeito deveria pressionar uma tecla do computador no momento em que aparecia o estímulo na tela do computador. Já a segunda condição abordou uma tarefa de memória de curto prazo, na qual o sujeito fazia a mesma tarefa da condição I e ao mesmo tempo deveria fazer uma contagem regressiva de dígitos. Os idosos saudáveis apresentaram um desempenho melhor na segunda condição de tarefa de memória de curto prazo, quando comparados aos pacientes com doença de Alzheimer. Comparando as duas condições, o grupo de pacientes com doença de Alzheimer apresentou pior desempenho na tarefa atencional.

Tabela 1. Características dos estudos que utilizaram métodos de avaliação de percepção de tempo em idosos com doença de Alzheimer

\begin{tabular}{lll}
\hline & Amostra/Caracterização & Avaliação cognitiva \\
& & \\
Papagno et al. & $\mathrm{n}=21$ pacientes com DA (71,86 anos) & MEEM, MODA \\
(2004) & $\mathrm{n}=21$ idosos saudáveis $(72,24$ anos) &
\end{tabular}

Rueda e SchmitterEdgecombe (2009)

MEEM, CDR, SDMT, RAVLT, BNT, WAIS III, DKEFS, GDS $\mathrm{n}=24$ idosos com CCL (70,96 anos) $\mathrm{n}=24$ idosos saudáveis (72,54 anos)

Experimento II $\mathrm{n}=17$ pacientes com DA (78,06 anos) $\mathrm{n}=17$ idosos saudáveis (76,65 anos)

Carrasco et al. (2000)

Caselli et al. (2009)

\section{$\mathrm{n}=8$ pacientes com DA $(62,13$ anos $)$} $\mathrm{n}=8$ idosos saudáveis (60 anos)

MEEM $\mathrm{n}=12$ pacientes com DA leve (70,6 anos) MEEM, MODA, ADL, VD, PAL $\mathrm{n}=12$ idosos saudáveis ( 71,4 anos) $\mathrm{n}=12$ jovens (28,4 anos)

\section{Métodos para avaliação de percepção de tempo}

Bissecção de tempo em duas condições: Condição l: tarefa atencional Condição Il: tarefa memória de curto prazo

Para ambas as condições: intervalos de duraçōes curtas (15 s) e durações longas (50 s)

\section{Bissecção de tempo em dois} experimentos: Experimento I: intervalos de durações curtas (10-25 s) e durações longas (40-60s)

Experimento Il: foi a mesma apresentada no experimento I

Bissecção de tempo no intervalo de durações curtas (5-10 s) e durações longas (25 s)

Bissecção de tempo no intervalo de durações curtas (100-600 ms) e durações longas (1.000-3.000 ms)

\section{Resultados}

Pacientes com DA apresentaram pior desempenho nas tarefas atencionais

Experimento I: os idosos com CCL apresentaram pior desempenho nas respostas de estimativa de tempo em durações curtas quando comparados aos idosos saudáveis

Experimento II: pacientes com DA subestimaram 0 tempo real quando comparados a idosos saudáveis

Pacientes com DA apresentaram dificuldade nas estimativas de tempo de durações longas

Pacientes com DA apresentaram pior desempenho na estimativa de tempo curto quando comparados a idosos saudáveis

ADL: Activities of Daily Living; BNT: Boston Naming Test; CCL:Comprometimento Cognitivo Leve; GDS: Geriatric Depression Scale; PAL: Paired Associate Learning; RAVLT: Rey Auditory-Verbal Learning Test; SDMT:Symbol Digit Modalities Test; VD: Visual Denomination; WAIS-III: Letter Number Sequencing subtest of the Wechsler Adult Intelligence Scale; DA: doença de Alzheimer. 


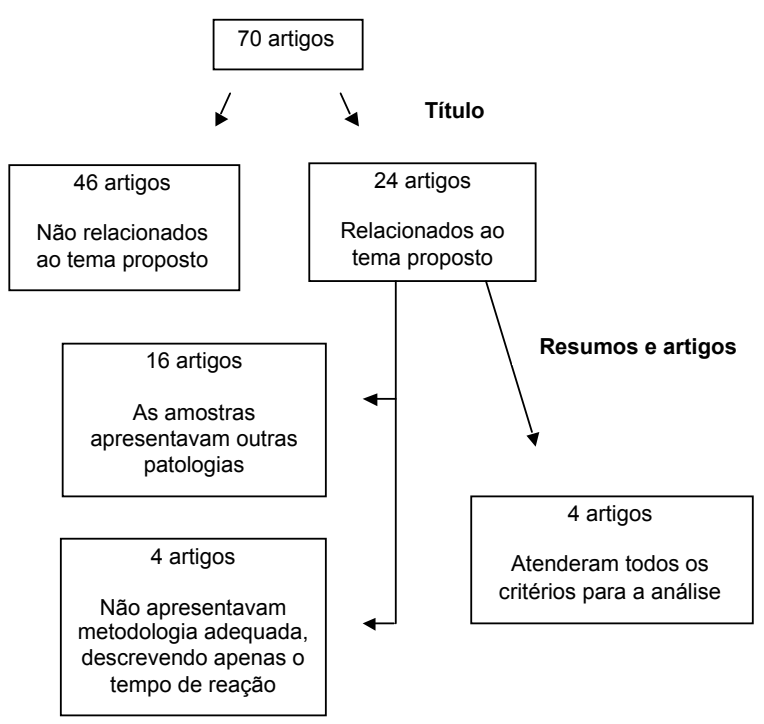

Figura 1. Etapas de seleção dos artigos incluídos na revisão sistemática.

2) Rueda e Schmitter-Edgecombe ${ }^{14}$ avaliaram a percepção de tempo nos intervalos de durações curtas (10-25 s) e longas (45-60 s) para a aplicação de dois experimentos. Para o experimento I, participaram 24 idosos com comprometimento cognitivo leve (média de idade de 70,96 anos; escolaridade média de 16,1 anos) com escore igual a 24 pontos no MEEM e 24 idosos saudáveis (média de idade de 72,54 anos; escolaridade média de 16,2 anos). O sujeito deveria pressionar uma tecla do computador no momento em que aparecia o estímulo-padrão e ao mesmo tempo deveria fazer uma contagem de dígitos. Os idosos com comprometimento cognitivo leve apresentaram pior desempenho nas respostas de estimativa de tempo curto quando comparados aos idosos saudáveis. No experimento II, participaram 17 pacientes com doença de Alzheimer (média de idade de 78,06 anos; escolaridade média de 14,82 anos) com escore menor que 24 no MEEM - 0,5 ponto na avaliação do Clinical Dementia Rating Scale (CDR) ${ }^{15}$ - e 17 idosos saudáveis (média de idade de 76,65 anos; escolaridade média de 15,76 anos). As tarefas foram as mesmas utilizadas no experimento I. Na tarefa de estimativa de tempo, os pacientes com doença de Alzheimer subestimaram o tempo real quando comparados ao grupo de idosos saudáveis.

3) Carrasco et al. ${ }^{16}$ avaliaram a percepção de tempo em uma amostra de 8 pacientes com doença de Alzheimer (média de idade de 62,13 anos; escolaridade média de 8,62 anos) com escore menor que 24 pontos no MEEM e 8 idosos saudáveis (média de idade de 60 anos; escolaridade média de 10,5 anos). A tarefa consistiu em dois sons, sendo intervalos de durações curtas (5-10 s) e durações longas ( 25 s), na qual os idosos tinham que diferenciar o som e responder a intervalos de durações (curtas e longas). Pacientes com doença de Alzheimer apresentaram pior desempenho em estimar o tempo de curta duração quando comparados aos idosos saudáveis.

4) Caselli et al. ${ }^{3}$ avaliaram a percepção de tempo em 12 pacientes com doença de Alzheimer leve (média de idade de 70,6 anos; escolaridade média de 5,7 anos) com escore menor que 22 pontos no MEEM e 83,09 pontos no Milan Overall Dementia Assessment (MODA) ${ }^{17}$ e 12 idosos saudáveis (média de idade de 71,4 anos; escolaridade média de 5 anos). Os participantes foram expostos aos intervalos de durações curtas (100-600 ms) e longas (1.000-3.000 ms). A tarefa consistiu em responder verbalmente em relação à percepção de tempo em que um estímulo permanecia na tela do computador. Pacientes com doença de Alzheimer apresentaram pior desempenho na estimativa de tempo curto quando comparados aos idosos saudáveis.

\section{DISCUSSÃO}

Do total de 70 artigos inicialmente examinados, quatro foram definitivamente elegíveis para o presente estudo. Todos os estudos demonstraram pior desempenho na estimativa de tempo de pacientes com doença de Alzheimer. Três estudos ${ }^{3,14,16}$ não descreveram as funções cognitivas nas quais se constatou pior desempenho na tarefa de bissecção em pacientes com doença de Alzheimer, entretanto o único que o fez foi o estudo de Papagno et al. ${ }^{12}$, verificando que pacientes com doença de Alzheimer apresentavam pior desempenho em tarefas que exigiam maior demanda atencional.

Em relação aos parâmetros utilizados no método de bissecção, foram notadas grandes variações entre os intervalos de durações "curtas" e "longas", o que dificulta padronizar ou mesmo elaborar parâmetros que sejam mais adequados para avaliar percepção de tempo em pacientes com doença de Alzheimer. Os resultados do estudo de Rueda e Schmitter-Edgecombe ${ }^{14}$, em uma tarefa de bissecção de tempo, na qual compararam a estimativa verbal nos intervalos de durações curtas (10-25 s) e longas (45-60 s), verificaram que pacientes com doença de Alzheimer subestimaram as durações de tempo longo.

Caselli et al. ${ }^{3}$ admitiram intervalos de durações curtas (100-600 ms) e longas (3.000 ms), em que pacientes com doença de Alzheimer exibiram sensibilidade para escalas de intervalos de durações curtas em tarefas atencionais. Esses resultados podem ser explicados pelo modelo Scalar Expectancy Theory $(\mathrm{SET})^{18}$, que avalia resultados de discriminação de tempo. Esse modelo aponta que quanto menor a atenção dispensada à percepção de tempo, menos pulsos irão chegar ao acumulador. A abertura do interruptor depende da atenção, e, como há menor quantidade de pulsos, a tendência é subestimar ${ }^{19}$. 
Esses achados corroboram os dados da literatura, os quais apontam que o baixo desempenho em tarefas de bissecção de tempo em pacientes com doença de Alzheimer pode ser explicado pelo fato de a atenção ser muito utilizada nessa condição ${ }^{20}$. Sabe-se que o curso clínico da doença de Alzheimer inicia-se pela alteração da memória e déficits na atenção, fazendo com que essa população apresente maior dificuldade na realização de tarefas que envolvam bissecção de tempo e discriminação nas variações de intervalos, nas durações curtas e longas 1,21,20. A partir desse achado, podemos inferir que o baixo desempenho na percepção de tempo em pacientes com doença de Alzheimer parece estar associado ao declínio das funções cognitivas, destacando a dificuldade de focar a atenção no estímulo dado.

Mimura et al. ${ }^{22}$ explicam o mecanismo de armazenamento de novas informações na memória, de modo que a informação antiga é removida mais cedo do que o normal, induzindo a sensação de que mais tempo passou, especialmente para intervalos de durações curtas. Esse mecanismo poderia explicar o fato de que pacientes com doença de Alzheimer apresentam uma grande superestimação para durações curtas, sugerindo que haja dificuldades na concentração para a realização da tarefa ${ }^{23}$.

Nichelli et al. ${ }^{20}$ realizaram um dos primeiros trabalhos envolvendo pacientes com doença de Alzheimer em tarefas de bissecção do tempo em intervalos de durações de 1 segundo. Os resultados mostraram grande variabilidade para essa população e tendência à superestimação. Carrasco et al.21 avaliaram a estimativa de tempo em pacientes com doença de Alzheimer empregando uma tarefa de estimativa de tempo para intervalos de durações curtas ( $5 \mathrm{~s}, 10 \mathrm{~s}$ ) e longas ( 25 s) e encontraram diferenças significativas no erro absoluto e na variabilidade em todos os intervalos.

Esses resultados sugerem que pacientes com doença de Alzheimer podem apresentar uma independência parcial entre duas medidas de estimativa de tempo (latência e erro absoluto). Assim, os resultados indicam que os escores de erro absoluto são mais sensíveis na detecção de decréscimos sutis no desempenho em tarefas de natureza tempo$\mathrm{ral}^{24,25}$. Por outro lado, a medida de variabilidade revela que os pacientes também são imprecisos em suas estimativas de tempo, sugerindo que as diferentes medidas de intervalo não concordam entre si. Assim, os resultados do estudo de Carrasco et al..$^{21}$ também mostraram que o tempo estimado pelos pacientes com doença de Alzheimer, em comparação com idosos saudáveis, é inconsistente como observado anteriormente por Nichelli et al.20.

Esses achados são contraditórios com as conclusões do estudo Killen e Fettermann ${ }^{26}$, que detectaram uma habilidade preservada para estimar diferentes intervalos de durações de tempo (12 s, 24 s, 60 s e 180 s), em pacientes com doença de Alzheimer. No estudo de Carrasco et al. ${ }^{21}$, a análise do tempo estimado não apresentou diferença entre os grupos de intervalo em durações de 10 e $25 \mathrm{~s}$. No entanto, quando os resultados são analisados considerando os escores de erro absoluto, parece que os pacientes com doença de Alzheimer apresentaram déficits na estimativa de intervalos de durações curtas. Nesse contexto, esses dados mostram a importância de analisar os escores de erro absoluto para uma melhor abrangência do desempenho em tarefas de estimativa de tempo em pacientes com doença de Alzheimer.

\section{CONCLUSÃO}

Este trabalho de revisão sistemática evidenciou que: a) pacientes com doença de Alzheimer apresentam prejuízos na tarefa de bissecção de tempo; esse fato pode ser explicado por a progressão da doença ser acompanhada por declínio gradual nas habilidades cognitivas, funcionais e psicomotoras, as quais são utilizadas no teste de percepção de tempo; b) há grandes variações nos intervalos de tempo utilizados no método em questão. Nesse contexto, há necessidade de mais estudos, controlados e randomizados, para investigar potenciais efeitos das variações nos intervalos de tempo no método de bissecção. Os resultados de tais estudos poderão contribuir para o estabelecimento de parâmetros mais adequados e fidedignos, na detecção de possíveis déficits de percepção de tempo em pacientes com doença de Alzheimer.

\section{AGRADECIMENTOS}

Laboratório de Atividade Física e Envelhecimento (Lafe), Grupo de Estudos: Aspectos Neuropsiquiátricos e Motricidade humana (GEANM).

\section{REFERÊNCIAS}

1. Sheridan PL, Solomont J, Kowall N, Hausdorff JM. Influence of executive function on I0comotor function: divided attention increases gait variability in Alzheimer's disease. J Am Geriatr Soc. 2003:51(11):1633-7.

2. James W. The principles of psychology. New York: Dover; 1980.

3. Caselli L, Laboli L, Nichelli P. Time estimation in mild Alzheimer's disease patients. Behav Brain Funct. 2009;5(32):1-10.

4. Lewis PA. Finding the timer. Trends Cogn Sci. 2002;6(5):195-6.

5. Buhusi CV, Meck WH. What makes us tick? Functional and neural mechanisms of interval timing. Nat Rev Neuroscie. 2005;6(10):755-65.

6. Block RA, Zakay D, Hancock PA. Human aging and duration judgments: a meta-analytic review. Psychol Aging. 1998;13(4):584-96.

7. Wittmann M. Time perception and temporal processing levels of the brain. Chronobiol Int. 1999;16(1):17-32.

8. Wearden JH, Jones LA. Is the growth of subjective time in humans a linear or nonlinear function of real time? Q J Exp Psychol. 2007;60(9):1289-302.

9. Stuart-Hamilton I. Psicologia do envelhecimento: uma introdução. São Paulo: Veronese; 2002. 
10. Brown SW. Time perception and attention: the effects of prospective versus retrospective paradigms and task demands on perceived duration. Percept Psychophys. 1985;38(2):115-24.

11. Folstein MF, Folstein SE, Mchugh PR. "Mini-Mental State". A practical method for grading the cognitive state of patients for the clinical. J Psychiatr Res. 1975;12(3):189-98.

12. Papagno C, Allegra A, Cardaci M. Time estimation in Alzheimer's disease and the role of the central executive. Brain Cogn. 2004;54(1):18-23.

13. Folstein MF, Folstein SE, Mchugh PR. Mini-mental state: a practical method for grading the cognitive state of patients for the clinician. J Psychiatric Res. 1975;12(3):189-98.

14. Rueda AD, Schmitter-Edgecombe M. Time estimation abilities in mild cognitive impairment and Alzheimer's disease. Neuropsychology. 2009;23(2):178-88.

15. Morris JC. The Clinical Dementia Rating (CDR): current version and scoring rules. Neurology. 1993;43(11):2412-4

16. Carrasco MC, Bernal MC, Redolat R. Time estimation and aging: a comparison between young and elderly adults. Int J Aging Hum Dev. 2001;52(2):91-101.

17. Brazzelli M, Capitani E, Della Sala SS, Spinnler H, Zuffi M. A neuropsychological instrument adding to the description of patients with suspected cortical dementia: The Milan Overall Dementia Assessment. J Neurol Neurosurg Psychiatry. 1994;57(12):1510-7.
18. Gibbon J, Church RM, Warren HM. Scalar timing in memory. Acad Sci. 1984;423(1):52-77.

19. Coelho M, Ferreira JJ, Dias B, Sampaio C, Pavão MI, Castro-Caldas A. Assessment of time perception: the effect of aging. J Int Neuropsychol Soc. 2004;10(3):332-41.

20. Nichelli P, Venneri A, Molinari M, Tavani F, Grafman J. Precision and accuracy of subjective time estimation in different memory disorders. Brain Res Cogn Brain Res. 1993;1(2):87-93.

21. Carrasco MC, Guillem MJ, Redolat R. Estimation of short temporal intervals in Alzheimer's disease. Exp Aging Res. 2000;26(2):139-51.

22. Mimura M, Kinsbourne $M, O^{\prime}$ Connor M. Time estimation by patients with frontal lesions and by Korsakoff amnesics. J Int Neuropsychol Soc. 2000;6(5):517-28.

23. Fortin C, Massé N. Expecting a break in time estimation: attentional time-sharing without concurrent processing. J Exp Psychol Hum Percept Perform. 2000;26(6):1788-96.

24. Goldstone S. The variability of temporal judgment in psychopathology. New York: Academic Press; 1975.

25. Shaw C, Aggleton JP. The ability of amnesic subjects to estimate time intervals. Neuropsychol. 1994;32(7):857-73.

26. Killen PR, Fetterman JG. A behavioral theory of timing. Psychol Rev. 1988;95(2):274-95. 